\title{
Enabling and Exploiting Self-similar Central Symmetry Formations
}

\author{
Giuliano Punzo, Philippos Karagiannakis, Derek J. Bennet, Malcolm Macdonald, Stephan \\ Weiss, Senior Member, IEEE
}

\begin{abstract}
In this work a formation flying based architecture is presented within the context of a distributed antenna array. An artificial potential function method is used to control the formation whereby deviation from an all-to-all interaction scheme and swarm shaping are enabled through a self-similar connection network. Introduction of an asymmetric term in the potential function formulation results in the emergence of structures with a central symmetry. The connection network then groups these identical structures through a hierarchical scheme. This produces a fractal shape which is considered for the first time as a distributed antenna array exploiting the recursive arrangement of its elements to augment performance. A 5-element Purina fractal is used as the base formation which is then replicated a number of times increasing the antenna-array aperture and resulting in a highly directional beam from a relatively low number of elements. Justifications are provided in support of the claimed benefits for distributed antenna arrays exploiting fractal geometries. The formation deployment is simulated in Earth orbit together with analytical proofs completing the arguments aimed to demonstrate feasibility of the concept and the advantages provided by grouping antenna elements into coherent structures.
\end{abstract}

\section{Index Terms}

Autonomous Formation Flight, Fractal, Artificial Potential Functions, Antenna Array, Hierarchical Network, Beamforming.

G. Punzo is with the Department of Mechanical and Aerospace Engineering, Advanced Space Concept Laboratory, University of Strathclyde, 75 Montrose Street, Glasgow, G1 1XJ, UK e-mail: giuliano.punzo@strath.ac.uk

P. Karagiannakis is with the Department of Electrical and Electronic Engineering, Centre for Excellence in Signal \& Image Processing, University of Strathclyde, 204 George Street, Glasgow, G1 1XW, UK e-mail: philipp.karagiannakis@strath.ac.uk

D. J. Bennet is with the Department of Mechanical and Aerospace Engineering, Advanced Space Concept Laboratory, University of Strathclyde, 75 Montrose Street, Glasgow, G1 1XJ, UK e-mail: derek.bennet@strath.ac.uk

M. Macdonald is with the Department of Mechanical and Aerospace Engineering, Advanced Space Concept Laboratory, University of Strathclyde, 75 Montrose Street, Glasgow, G1 1XJ, UK e-mail: malcolm.macdonald102@strath.ac.uk

S. Weiss is with the Department of Electrical and Electronic Engineering, Centre for Excellence in Signal \& Image Processing, University of Strathclyde, 204 George Street, Glasgow, G1 1XW, UK e-mail: stephan.weiss@eee.strath.ac.uk

Manuscript received January 26, 2012; 


\section{INTRODUCTION}

The value of exploiting formation flight techniques for space science, remote sensing and telecommunications applications is gaining popularity [1]-[5]. So far proposed formation flying concepts have been based on a relatively low number of cooperating spacecraft, as in the case of Lisa, Proba-3 or StarLight missions [6]-[8]. The exploitation of a formation flight architecture with an increased number of elements which maintains an acceptable level of system complexity can be pursued through the control of autonomous and independent agents as a single group entity [2], [9].

Coupling reliable formation flying capabilities with the possibility of producing complex patterns using spacecraft will enable the potential of grouping a number of antenna elements into a cooperative structure. This has long been known and applied in antenna array theory [10], [11] and proposed at conceptual level for space applications [12][14].

The key point in the exploitation of formation flying techniques for the deployment of an antenna array is that the performance of a homogeneous pattern of array elements can be matched or surpassed by fractal geometries as per [15] and [16]. Fractal geometries as defined by [15] can be considered self-similar structures propagated from a core initiator through a number of stages of growth by an identical generator. Application of fractal geometries in antenna array design has mainly focussed on single structures, that is to say one device housing the antenna array. In this context each satellite houses an antenna which contributes to form the fractal pattern. Hence, the problem turns into producing a fractal pattern from a formation of spacecraft which provides a platform for a number of array elements able to exploit the fractal pattern characteristics.

From a control point of view this can be realised through artificial potential functions (APFs) which represent a popular control method particularly suited to large structures of autonomous agents, such as discussed in e.g. [17][19]. The way to obtain complex formations through APFs, while maintaining a high degree of reliability and analytically provable characteristics, can be revealed through the design of a limited connection network. Network characteristics reflect on the final pattern deployed through APF acting along its edges. In particular when the connection network presents self-similarity characteristics, i.e. the same network structure repeats for nodes and groups of nodes, this impacts not only on the final formation but also on the stability and robustness properties which are the same when considering the control of single spacecraft or groups of those. As consequence the overall control architecture result is scalable and possesses a certain degree of fault tolerance.

From the array point of view, self-similarity and sparseness lead to a number of benefits — similar performance in operation across a number of frequencies becomes possible due to the repetitive nature of the array pattern as per [15] and [16], array performance degrades gracefully with element failure and finally equivalent performance can be achieved for a fraction of the number of elements used in square lattice arranged arrays [20].

This paper proposes the deployment of a distributed fractal antenna array across a large group of satellites. Previous works, [14] and [13], have discussed the benefits of flying an arbitrary formation of distributed antenna elements to take advantage of the lower risks and costs associated with a network compared to a single large element. On the 
other hand there are examples in literature that investigate the benefits of a fractal shaped monolithic antenna [15]. The present work merges for the first time the concepts of distributed antenna arrays, fractal antennas and formation flying. The inherent control complexity is reduced through joint control techniques making use of APF and a selfsimilar communication network. In a similar fashion the overall antenna gain and performance is increased, even though when compared to a similar performing planar structure a reduced set of radiating elements is used.

A description of the theoretical background is provided in Sec. II and is followed by a more detailed mathematical analysis related to the specific problem in Sec. III. The topics covered include: the control method in terms of the APF characteristics and communication network; as well as an overview of fractal antenna theory, its application to a specific geometry and the resulting performance. In Sec. IV numerical simulations are performed for the case of an architecture in geostationary orbit although the set of equation used is valid in general for circular orbits and nothing prevents the concept from being applied to any other orbit. Discussion and Conclusions follow in Sec. V and Sec. VI respectively. This paper demonstrates the potential of implementing an innovative architecture based on multiple autonomous spacecraft forming a fractal array.

Notation. In this paper, vectors and matrices are denoted by lowercase and uppercase bold face variables, respectively. For two vectors $\mathbf{x}$ and $\mathbf{y}, \mathbf{x} \cdot \mathbf{y}$ is the scalar product. The first and second derivatives of a function $x$ with respect to time are, respectively, denoted by $\dot{x}$ and $\ddot{x}$. Finally, a linear approximation of a function $f$ at a given point is represented by $\tilde{f}$.

\section{Methodology}

A group of $N$ spacecraft is considered, divided into subgroups of $n$ agents such that $N=n^{k}$ with $k \in \mathbb{N}^{+}$. It is assumed that each spacecraft carries an element of the array where the pair spacecraft-array element will be named from here on as agent. Spacecraft and array element will instead be used when referring to these components of the complete system. The agents are connected according to a non directional graph described by an adjacency matrix $\mathbf{A} \in \mathbb{N}^{N \times N}$ containing binary elements $a_{i j}$, with $i, j \in[1, N]$. The spacecraft are controlled through pairwise APFs which act only along the edges of the graph. There is no global position or orientation of the agent formation, but within the formation, relative positions are considered for agents and groups of agents while relative orientation is considered for groups of agents only. This implies that the single array elements are pointed correctly or, as assumed here, are isotropic sources.

This section shows how a self-similar formation can be obtained from mutually interacting agents, and how the array performance can be analysed for such a system. For this purpose artificial potential function characteristics and communication graph topology are described. The fundamental concept of applying fractal geometries to the design of antenna arrays using a self-scaling method is described for the case of planar configurations only, although similar arguments can be applied to linear and 3D formations. 


\section{A. Artificial Potential Functions}

The spacecraft are controlled through artificial potential functions (APFs) operating along the edges of a communication network. The APFs operate on a pairwise basis, that is they do not depend on position or velocity of the agents but only on their state relative to the other spacecraft with which they are connected; in particular the Morse potential is used. This is composed of an attractive component

$$
U_{i j}^{a}=-C_{i j}^{a} \exp \left(-\frac{\left|\mathbf{x}_{i j}\right|}{L_{i j}^{a}}\right)
$$

and a repulsive component

$$
U_{i j}^{r}=C_{i j}^{r} \exp \left(-\frac{\left|\mathbf{x}_{i j}\right|}{L_{i j}^{r}}\right),
$$

where $C_{i j}^{a}$ and $C_{i j}^{r}$ are constants regulating the magnitude of the potential, while $L_{i j}^{a}$ and $L_{i j}^{r}$ are constants related to the attractive and repulsive scale lengths. The subscripts $i, j$ refer to the potential sensed by agent $i$ because of interaction with agent $j$. The relative position vector of agent $i$ with respect to agent $j$ is denoted by $\mathbf{x}_{i j}$. The control law is completed by a virtual viscous-like damping in the form $\sigma \mathbf{v}_{i}$, with $\sigma$ being a positive damping constant to be defined later and $\mathbf{v}_{i}$ representing agent velocity. This control law together with the hypothesis of no external disturbances and idealised sensing and actuation capabilities results in the motion equations

$$
\begin{aligned}
\dot{\mathbf{x}}_{i} & =\mathbf{v}_{i} \\
m \dot{\mathbf{v}}_{i} & =-\nabla U_{i}^{a}-\nabla U_{i}^{r}-\sigma \mathbf{v}_{i},
\end{aligned}
$$

where $m$ defines the agent mass and is assumed the same for all agents, and

$$
\begin{gathered}
\nabla(\cdot)=\frac{\partial(\cdot)}{\partial \mathbf{x}_{i}} \\
U_{i}^{a}=\sum_{j}\left(a_{i j} U_{i j}^{a}\right) \quad \text { and } \quad U_{i}^{r}=\sum_{j}\left(a_{i j} U_{i j}^{r}\right),
\end{gathered}
$$

with $a_{i j}$ being the entry of the adjacency matrix to be defined next.

\section{B. Adjacency Matrix}

As reported in Sec. II-A, agents communicate through a network of links. In general in a network system studied through graph theory an adjacency matrix contains non-zero entries in the $(i, j)$ location whenever there is a directed edge from node $i$ to node $j$, indicating a communication link between the two agents represented by these nodes. Moreover the matrix is not weighted, i.e. the elements $a_{i j} \in\{0,1\}$ are binary. The strength of the interactions is provided by the APF via (6). While the proposed adjacency matrix is symmetric, i.e. the graph is not directed, this does not imply that the virtual interactions amongst the agents are symmetric.

Within the adjacency matrix $\mathbf{A}$ for a system with $N=n^{k}$ agents, the edges belonging to fully connected $n$-agent subgroups form $n \times n$ submatrices along the block-diagonal. The remainder of the matrix contains links between agents in the $n^{k-1}$ different subgroups. 
Example 1. For the case $n=5$ and $k=2$, there are 5 subgroups creating $5 \times 5$ submatrices along the diagonal of the adjacency matrix, as indicated in Fig. 1. The communication between any pair of subgroups is maintained through one linking agent per subgroup (the central one), accounting for $n-1$ connections each. Beside that relative orientation of peripheral subgroups with respect to the central one is ensured by 1 linking agent per peripheral subgroup connecting to the adjacent one in the central core.

Example 2. For the case $n=5$ and $k=3$, there are 25 subgroups creating $5 \times 5$ submatrices along the diagonal of the adjacency matrix. These ones are connected in groups of 5 as described in Example 1, and are represented by the $25 \times 25$ squares along the diagonal of Fig. 2 . The communication between any pair of 25 -agent subgroups is this time ensured by groups of 5 agents that replace the single agents of Example 1.

The network is designed such that the peripheral nodes are weaker than the central ones. This means that loss of control of one node due to loss of link is more likely for nodes that belong to peripheral region of the formation, hence they do not play as bridge between large portions of the ensemble. This implies that the loss of some links is more likely to produce the disconnection of a smaller and peripheral portions of the network than of a large portion. Each node is in any case at least connected to $n-1$ other nodes. When the number of generators increases, those groups which were end-points for the previous generator become embedded and more firmly bonded into the larger pattern. This ensures that in the most critical scenario the loss of at least $n-1$ links is needed for fragmentation to occur. In Fig. 3 the node degree is reported for the adjacency matrix of dimension 125, that is the number of links each node is connected to. Nodes are sorted from the central to the peripheral ones.

\section{Fractal Electrodynamics}

Based on the above control methods to shape a group of agents into a fractal geometry, this section addresses their performance as an antenna. This is assessed by means of fractal electrodynamics, which is defined as the combined study of fractal geometries with electromagnetic theory and provides methods for the theoretical analysis and synthesis of fractal antenna arrays. One of the key metrics used to evaluate antenna array performance is directivity - it defines how the power radiated varies as a function of the angle of arrival when observed in the antenna far field. Utilising the methods described in this section, specific fractal geometries and their directivity will be addressed in Sec. III-D.

Since the focus of this work lies in the control of two-dimensional planar structures, only the design and analysis of planar fractal antenna arrays is described. As the proposed fractal antenna array is part of a satellite constellation whose aperture is small when compared to its orbit, Cartesian coordinates are used to describe it. Directivity, which is generally derived from the product of the array factor [10] and the radiation characteristics of the individual antenna array elements, is here only dependent on the array factor since isotropic antenna elements are assumed. The array factor is a function of the geometry of the array and the excitation phase. Varying the separation and/or phase between the antenna elements allows the total field of the array to be controlled and alters the characteristics of the array factor. Fig. 4 shows a symmetric planar array with uniformly spaced elements, separated by distances $d_{x}$ and $d_{y}$ in the $x$ - and $y$-directions. The array factor for such a symmetric planar array configuration has been 


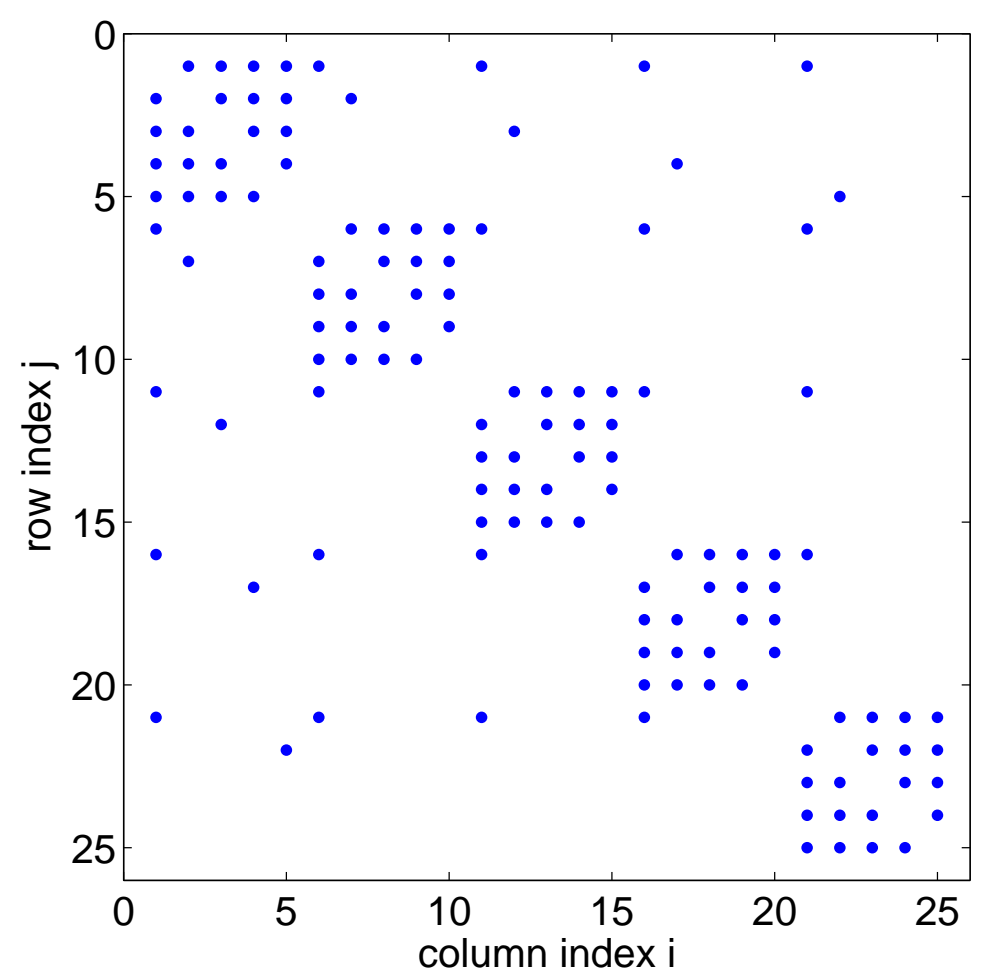

Fig. 1. Adjacency matrix for the case $n=5$ and $k=2$, creating a group of $N=25$ agents. Non-zero entries are represented by dots.

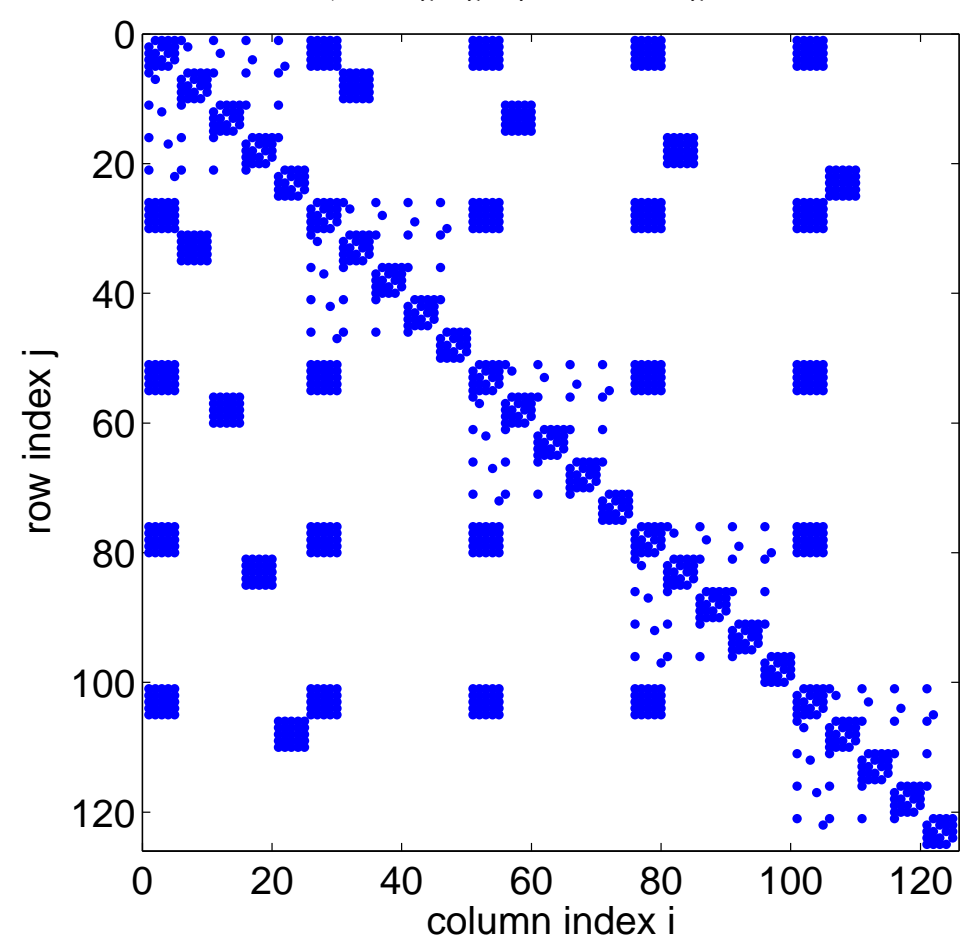

Fig. 2. Adjacency matrix for the case $n=5$ and $k=3$, creating a group of $N=125$ agents. The self-similarity of the matrix can be observed. The 25-agent matrix of Fig. 1 is replicated now 5 times along the diagonal and the other entries of the matrix, grouped in $5 \times 5$ squares are in the same positions as the links in the 25-agent matrix. 


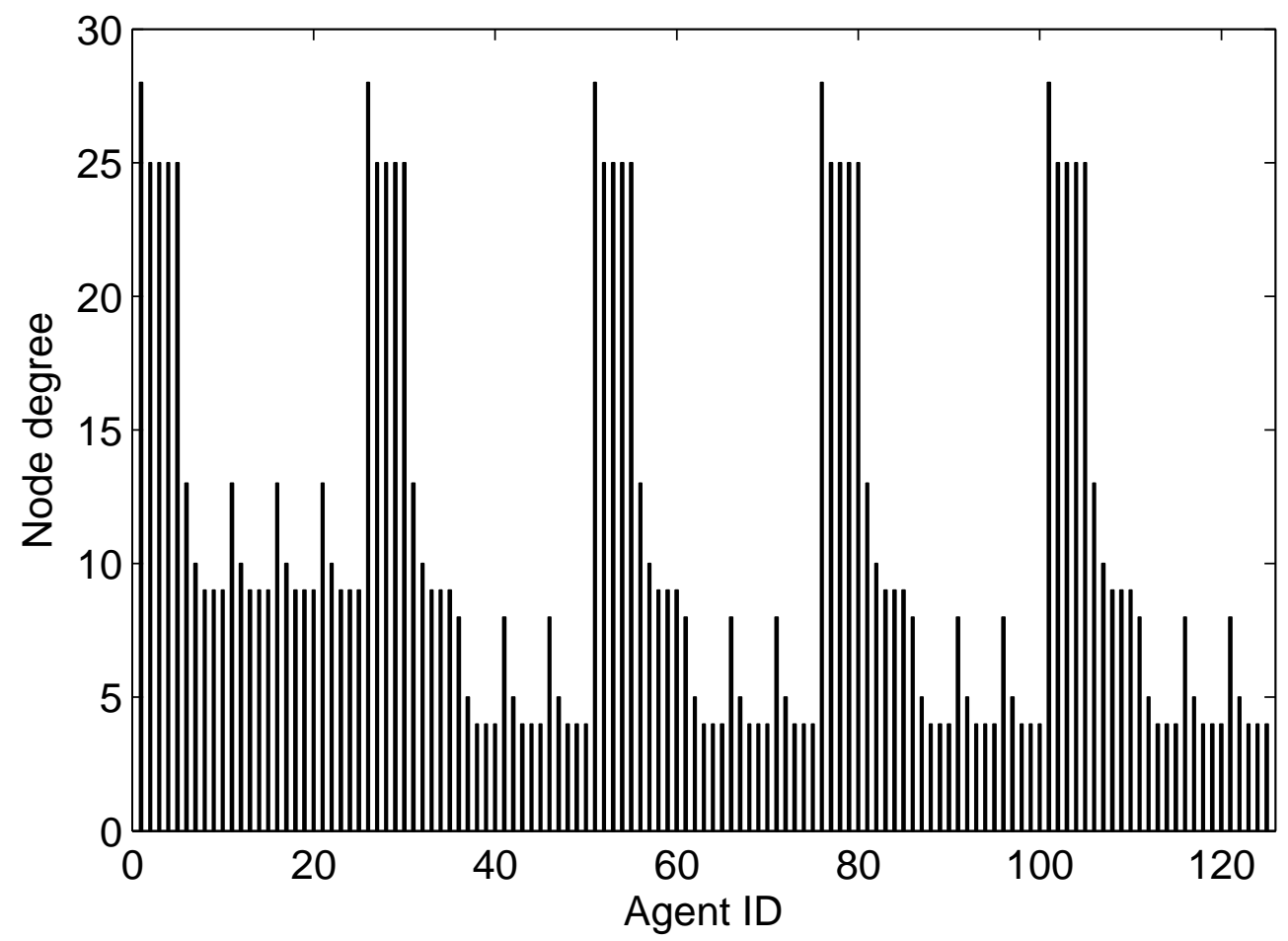

Fig. 3. Node degrees as number of links belonging to each node. A self-similar scheme can be observed with nodes in central position being the most connected ones. In this scheme the maximum number of connections per node is 28 .

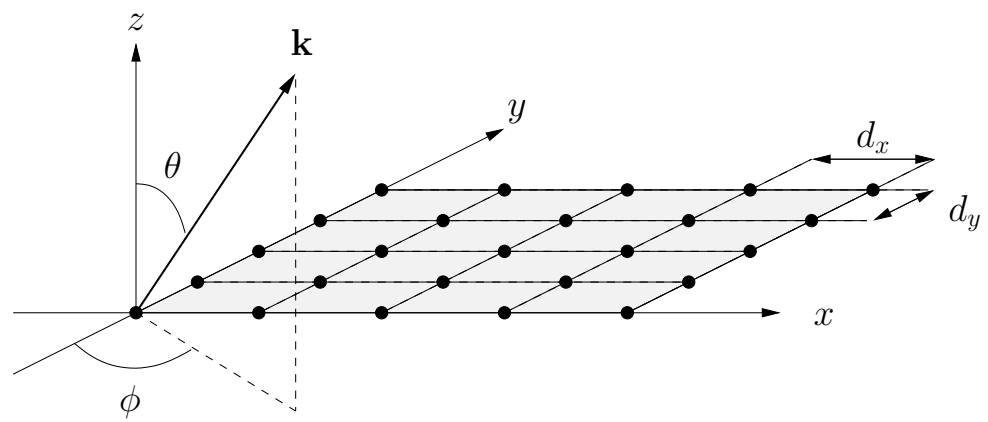

Fig. 4. Symmetric planar array in the $x-y$ plane with inter-element spacings of $d_{x}$ and $d_{y}$, and definition of spherical angles $\theta$ and $\phi$ for the wavenumber vector $\mathbf{k}$ of a farfield source.

derived as [10] based on the weighting $S_{m n}$ of fractal elements,

$$
\Gamma\left(u_{x}, u_{y}\right)= \begin{cases}S_{11}+2 \sum_{m=2}^{M}\left(S_{m 1} \cos \left(m u_{x}\right)+S_{1 m} \cos \left(m u_{y}\right)+2 \sum_{n=2}^{N} S_{m n} \cos \left(m u_{x}\right) \cos \left(n u_{y}\right)\right) & , M \text { odd } \\ 4 \sum_{n=1}^{N} \sum_{m=1}^{M} S_{m n} \cos \left(\left(m-\frac{1}{2}\right) u_{x}\right) \cos \left(\left(n-\frac{1}{2}\right) u_{y}\right) & M \text { even, }\end{cases}
$$

whereby the array factor $\Gamma\left(u_{x}, u_{y}\right)$ is expressed in dependency of a wavenumber $\mathbf{k}$ projected onto the $x-y$ plane 
of the array, giving projection lengths weighted by the inter-element spacings $d_{x}$ and $d_{y}$,

$$
\begin{aligned}
& u_{x}=d_{x}|\mathbf{k}|\left(\sin \theta \cos \phi-\sin \theta_{0} \cos \phi_{0}\right) \\
& u_{y}=d_{y}|\mathbf{k}|\left(\sin \theta \sin \phi-\sin \theta_{0} \sin \phi_{0}\right) .
\end{aligned}
$$

In (8) and (9), $\theta$ and $\phi$ are the angle of incident of a potential source illuminating the array, $|\mathbf{k}|=\frac{2 \pi}{\lambda}$ the modulus of the wavenumber depending on the wavelength $\lambda$ of the source, and $\theta_{0}$ and $\phi_{0}$ define the look direction of the array.

Deterministic fractal arrays are constructed in a self-similar manner and consist of many smaller parts whose shape resembles that of the overall object. They are formed by the repetition of a generating sub-array at scale one; to construct higher scales of growth, repetitions of this small sub-array are used. Utilising (7) and the methodology defined above, it is possible to construct and analyse a deterministic planar fractal array. The pattern of the generating sub-array is achieved by switching elements of a fully populated symmetric array on or off according to

$$
S_{m n}=\left\{\begin{array}{ll}
1, & \text { if element }(m, n) \text { is turned on } \\
0, & \text { if element }(m, n) \text { is turned off }
\end{array},\right.
$$

until the desired fractal pattern emerges.

Following (10), the thinned generating sub-array can be copied, scaled and translated to produce the final array. Due to the recursive nature of the development procedure, deterministic fractal arrays created in this manner can conveniently be thought of as arrays of arrays. The array factor for a deterministic fractal array may be expressed in a general form given as a product of scaled versions of the same generating sub-array pattern [15],

$$
\Gamma_{P}(\mathbf{u})=\prod_{p=1}^{P} \Gamma_{\text {frac }}\left(\delta^{p-1} \mathbf{u}\right)
$$

where $\Gamma_{P}(\mathbf{u})$ represents the array factor of the fractal generating sub-array resulting from the thinning of the symmetric planar array, and $\mathbf{u}$ is the vector of dependent variables. The expansion factor $\delta$ controls how much the array grows with each application of the generating sub-array and is inherited from the size of the symmetric planar array prior to thinning. Further, the parameter $P$ in (11) represents the scaling level/growth stage.

The directivity $D(\theta, \phi)$ of an array measures the power radiated in a specific direction defined by spherical angles $\theta$ and $\phi$, such as for the planar case in Fig. 4. While for single-element antennas the electrical and physical dimensions require adjustment to achieve a steering towards specified directions, grouping individual antenna elements into arrays can enable highly directional radiation pattern. For a planar antenna array with a symmetrical radiation pattern, the directivity is given by

$$
D(\theta, \phi)=\frac{F^{2}(\theta, \phi)}{\frac{1}{4 \pi} \int_{0}^{2 \pi} \int_{0}^{\pi} F^{2}(\vartheta, \varphi) \sin (\vartheta) d \vartheta d \varphi},
$$

where $F(\theta, \phi)$ denotes the radiation intensity of the antenna in the direction of the angles $\theta$ and $\phi$. For the case of isotropic sources, where individual antenna radiation patterns are unity and the radiation intensity reduces to the antenna array factor $\Gamma_{P}(\theta, \phi)$ in $(11)$, and $F(\theta, \phi)=\Gamma_{p}(\theta, \phi)$ can be substituted into (12). 
By grouping a number of smaller distributed antenna elements it is possible to form a fractal antenna array. The design and analysis of a fractal antenna array has been described above and provides the possibility to increase directivity and steer the main beam of an antenna. This offers a number of benefits in the context of space communications, including cost reduction and risk mitigation due to reduced number of antenna array elements. Fractal arrays evaluated following the procedure detailed above belong to a special category of thinned arrays. Application of the pattern multiplication theorem to the analysis and design of planar fractal arrays is considered in subsequent sections.

\section{Control Law and Fractal Antenna Analysis}

In this section the characteristics of the control technique used to drive an ensemble of agents towards the formation of a fractal pattern and the issues related to the design of a fractal shaped antenna array are considered. It is first shown how asymmetry in attraction-repulsion potential leads necessarily to a central symmetry configuration. It is then shown how the APF coefficients are calculated in order to get the desired distance between agents. Analysis of the control law is completed by considering the nonlinear stability characteristics. Fractal antenna design methodology is finally illustrated in detail for the case of a Purina fractal antenna array [15]. With reference to Sec. II, from now on only the case of an initiator of $n=5$ elements is considered.

\section{A. Central Symmetry Emergence}

Central symmetry emerges at initiator level by means of asymmetry between the interactions of one single agent with the group. This is obtained through a different value of the $L_{i j}^{r}$ parameter along the directed edges connecting the agent to the other 4 in the initiator structure. This is here explained by finding the conditions that make the artificial potential derivatives null along two orthogonal axes which are centred on the agent considered and define the plane where the control is exerted. The out of plane motion is undertaken through other means and is explained in Sec. IV. Considering the 5-agent scheme, given in Fig. 5, the first derivative of the artificial potential sensed by agent 1 can be calculated for the regular pentagon formation pictured. Then the conditions that apply to the APF coefficients in order to reach a stable equilibrium are deduced. APF derivatives can be calculated as

$$
\begin{aligned}
& \frac{\partial U_{i}}{\partial x_{i}}=\sum_{j=1}^{n}\left(\frac{C_{i j}^{a}}{L_{i j}^{a}} \exp \left(-\frac{\left|\mathbf{x}_{i}-\mathbf{x}_{j}\right|}{L_{i j}^{a}}\right)-\frac{C_{i j}^{r}}{L_{i j}^{r}} \exp \left(-\frac{\left|\mathbf{x}_{i}-\mathbf{x}_{j}\right|}{L_{i j}^{r}}\right)\right) \frac{x_{i}-x_{j}}{\left|\mathbf{x}_{i}-\mathbf{x}_{j}\right|} \\
& \frac{\partial U_{i}}{\partial y_{i}}=\sum_{j=1}^{n}\left(\frac{C_{i j}^{a}}{L_{i j}^{a}} \exp \left(-\frac{\left|\mathbf{x}_{i}-\mathbf{x}_{j}\right|}{L_{i j}^{a}}\right)-\frac{C_{i j}^{r}}{L_{i j}^{r}} \exp \left(-\frac{\left|\mathbf{x}_{i}-\mathbf{x}_{j}\right|}{L_{i j}^{r}}\right)\right) \frac{y_{i}-y_{j}}{\left|\mathbf{x}_{i}-\mathbf{x}_{j}\right|}
\end{aligned}
$$

with $U_{i}=U_{i}^{a}+U_{i}^{r}$. Excluding the trivial case for $L_{i j}^{r}=L_{i j}^{a}$ and $C_{i j}^{r}=C_{i j}^{a}$, (13) and (14) can be driven to zero while satisfying the stability condition $L_{i j}^{r}<L_{i j}^{a}$ [18]. From here on, just changes in $L_{i j}^{r}$ are considered, where $i, j$ refers to the indexing within the 5 agent group. In contrast, $L_{i j}^{a}, C_{i j}^{a}$ and $C_{i j}^{r}$ are considered independent from the pair of agents i.e. they take the same value for every index $i, j$ and will hence be omitted below.

Taking the planar formation in Fig. 5, the equilibrium along $y$ is trivially satisfied for all possible distances $d$ either in case $L_{i j}^{r}=L^{r}$ for all $(i, j)$, that is it takes the same values along all the edges, or in the case one agent has 


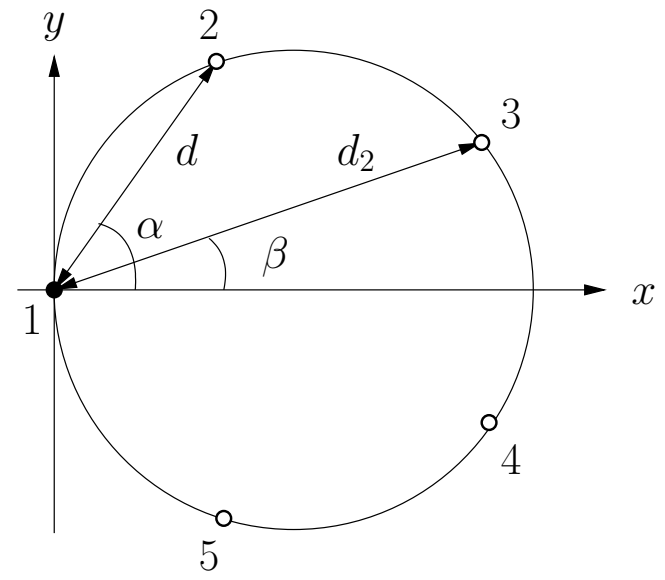

Fig. 5. Configuration with 5 agents — all having APFs with identical coefficients — arranged in a homogeneous formation.

a different repulsive scale distance. This can be understood by simply considering the symmetry of the formation about $x$-axis. Equilibrium along the $x$-axis does not lead to an explicit expression for the equilibrium distance, nonetheless the derivative of the potential w.r.t. $x$ referring to any agent can be calculated. Due to the homogeneity of the configuration any agent can be taken to analyse the artificial potential field. In particular for agent 1 ,

$$
\begin{aligned}
\left.\frac{\partial U_{1}}{\partial x_{1}}\right|_{\text {pent. }=2 \frac{C^{a}}{L^{a}}} & \left(\exp \left(-\frac{d}{L^{a}}\right) \cos \alpha+\exp \left(-\frac{d_{2}}{L^{a}}\right) \cos \beta\right) \\
& -2 \frac{C^{r}}{L^{r^{\prime}}}\left(\exp \left(-\frac{d}{L^{r^{\prime}}}\right) \cos \alpha+\exp \left(-\frac{d_{2}}{L^{r^{\prime}}}\right) \cos \beta\right),
\end{aligned}
$$

where

$$
d_{2}=\frac{d}{2} \sqrt{\left(\tan \alpha+\frac{1}{\cos \alpha}\right)^{2}+1}=k d
$$

can be determined, with $k>1$. This is considered as an initial equilibrium scenario for some equilibrium distance $d$ and for $L^{r}=L^{r^{\prime}}$ that is the same repulsive scale distance sensed by all the agents. In this scenario (15) must equal zero, but if $L^{r} \neq L^{r \prime}$ and in particular $L^{r}<L^{r^{\prime}}$ the separation distance must shrink. Thus the equilibrium distance reduces as the scale separation distance shrinks. This can be verified by differentiating (15) w.r.t. $L^{r \prime}$, leading to

$$
\begin{aligned}
\left.\frac{\partial^{2} U_{1}}{\partial x_{1} \partial L^{r^{\prime}}}\right|_{\text {pent. }}= & 2 \frac{C^{r}}{L^{r^{\prime 2}}}\left(\exp \left(-\frac{d}{L^{r^{\prime}}}\right) \cos \alpha+\exp \left(-\frac{k d}{L^{r^{\prime}}}\right) \cos \beta\right. \\
& \left.-\frac{d}{L^{r^{\prime}}} \exp \left(-\frac{d}{L^{r^{\prime}}}\right) \cos \alpha-\frac{k d}{L^{r^{\prime}}} \exp \left(-\frac{k d}{L^{r^{\prime}}}\right) \cos \beta\right) .
\end{aligned}
$$

The expression in (17) is negative definite, since a reduction of $L^{r^{\prime}}$ produces an acceleration on agent 1 in the direction of the positive $x$-axis and therefore leads to a reduction of its equilibrium distance,

$$
\begin{aligned}
& \left.\frac{\partial^{2} U_{1}}{\partial x_{1} \partial L^{r^{\prime}}}\right|_{\text {pent. }}<0 \\
\therefore \quad & \left(1-\frac{d}{L^{r^{\prime}}}\right)\left(\exp \left(-\frac{d}{L^{r^{\prime}}}\right) \cos \alpha\right)+\left(1-\frac{k d}{L^{r^{\prime}}}\right)\left(\exp \left(-\frac{k d}{L^{r^{\prime}}}\right) \cos \beta\right)<0 .
\end{aligned}
$$




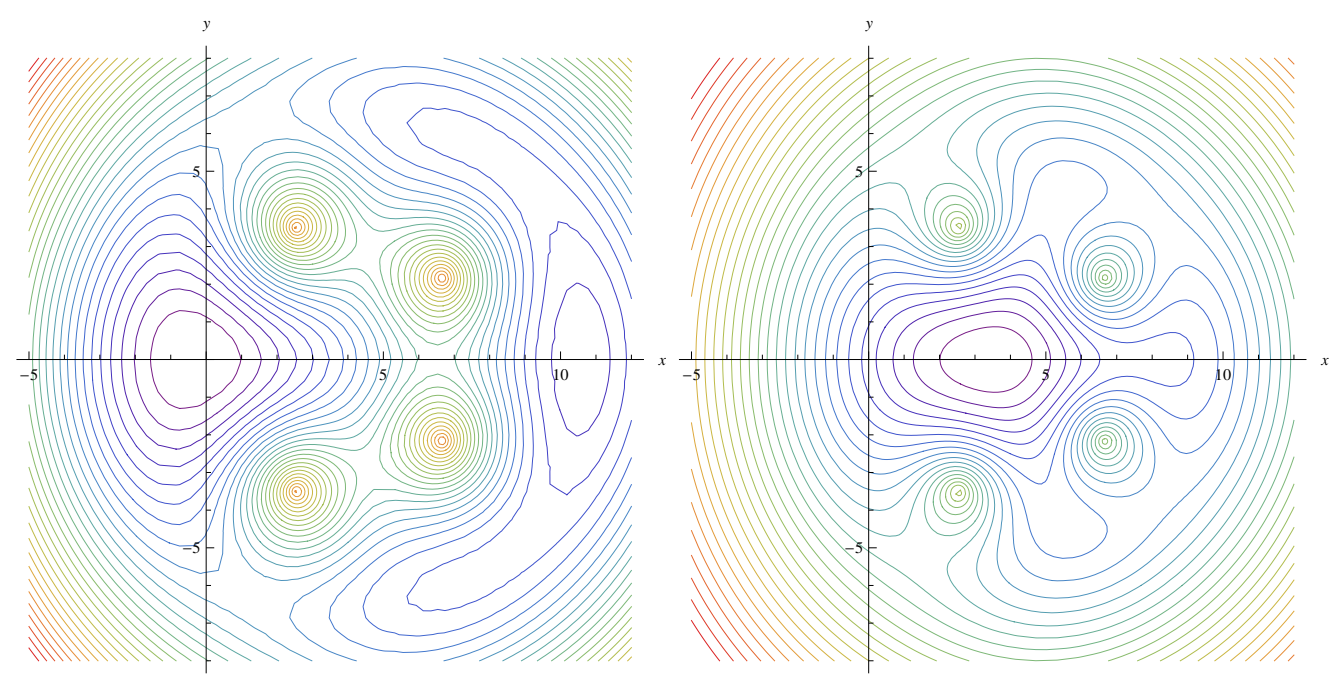

(a)

(b)

Fig. 6. Contours for the potential sensed by an agent at the origin (a) in the case all agents have the same value of the repulsive potential scale length $L^{r}$ and (b) in the case the central agent has a repulsive scale distance $L^{r \prime}<L^{r}$.

This is always satisfied for $d>L^{r^{\prime}}$. The sufficient condition $d>L^{r \prime}$ can be obtained by a wide choice of system parameters, which can be easily seen by inspecting the equilibrium distance for the simple case of two agents. This case is obtained by summing up and setting equal to zero the derivatives in (1) and (2) for $\left|\mathbf{x}_{i j}\right|_{2}=d$, and then solving for $d$,

$$
d=\frac{L^{a} L^{r}}{L^{r}-L^{a}} \ln \frac{C^{a} L^{r}}{C^{r} L^{a}}>L^{r}
$$

In particular for $C^{a}=C^{r}$ the relationship shown in (19) is true as long as $L^{a} \neq L^{r}$. However, as stability imposes $L^{a}>L^{r}$, to make the potential function convex in the vicinity of the equilibrium, it can be concluded that (19) is always verified for stable potentials and possible to achieve for other choices of the parameters $C^{a}$ and $C^{r}$.

The other agents in the group considered in Fig. 5 tend to keep the same relative distance w.r.t. agent 1. This produces the new equilibrium configuration that sees the agent with reduced separation distance finding its equilibrium position in the centre of the 5-agent group while fulfilling also equilibrium conditions for the other agents. A contour plot of the potential which agent 1 senses is reported in Fig. 6 for both equilibrium and nonequilibrium parameter choices.

By similarly working the $C^{r}$ parameter, the same effect can be obtained as (15) is linear in $C^{r}$. Here, parameter $L^{r^{\prime}}$ is used to force the central symmetry configuration over the pentagon one, while parameter $C^{r}$ is used to produce the desired inter-agent distance only. The cross configuration generated by the asymmetry in the potential repulsive scale length is sketched in Fig. 7.

Considering that interactions amongst agents are only along the edges of the adjacency matrix, a representation of the repulsive and attractive scale parameter as well as of the other coefficients influencing (1) and (2) can be given in terms of matrix which have the same structure of the adjacency matrix described in Sec. II-B. An extract 


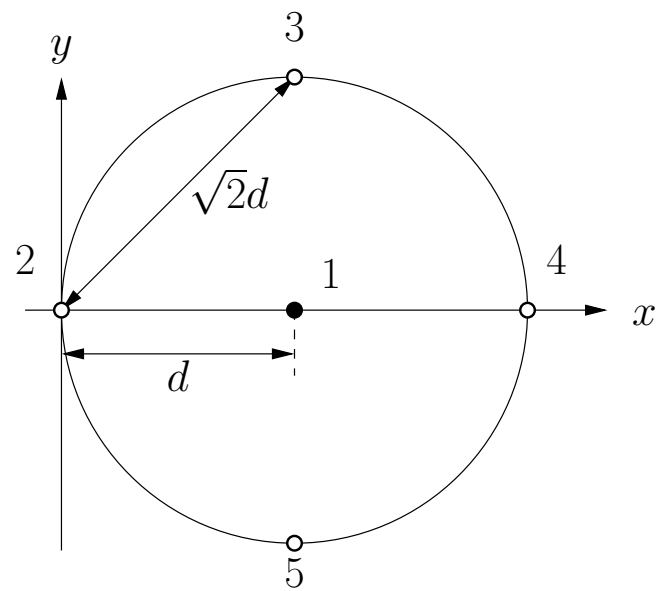

Fig. 7. Cross pattern emerging by shrinking the repulsive potential scale length sensed by the agent in the centre.

from the top left-hand corner of the repulsive distance matrix is given by

$$
\left[\begin{array}{ccccccccc}
0 & L^{r} & L^{r} & L^{r} & L^{r} & L_{2}^{r} & 0 & 0 & \\
L^{r \prime} & 0 & L^{r} & L^{r} & L^{r} & 0 & L_{3}^{r} & 0 & \\
L^{r \prime} & L^{r} & 0 & L^{r} & L^{r} & 0 & 0 & 0 & \\
L^{r^{\prime}} & L^{r} & L^{r} & 0 & L^{r} & 0 & 0 & 0 & \cdots \\
L^{r^{\prime}} & L^{r} & L^{r} & L^{r} & 0 & 0 & 0 & 0 & \\
L_{2}^{r^{\prime}} & 0 & 0 & 0 & 0 & 0 & L^{r} & L^{r} & \\
0 & L_{3}^{r} & 0 & 0 & 0 & L^{r^{\prime}} & 0 & L^{r} & \\
0 & 0 & 0 & 0 & 0 & L^{r^{\prime}} & L^{r} & 0 & \\
& & & \vdots & & & & & \ddots
\end{array}\right]
$$

where zeros are in the same positions as in the adjacency matrix in Figs. 1 and 2, and where the coefficients regulating the interactions among nodes which are centres of two different 5-agent groups are denoted by $L_{2}^{r}$. Finally $L_{3}^{r}$ is used to indicate the value along the edges connecting peripheral agents across different 5-agent groups. Hence coefficients $L^{r}, L^{a}, C^{r}$ and $C^{a}$ can be arranged in square matrices of dimension $N$; as these coefficients refer to the edges of the graph, they take a different value depending on which agent the edge is connected to.

One consideration which is worth noting is that arrangement in pentagon configuration is not guaranteed by the condition $L^{r \prime}=L^{r}$. While having $L^{r^{\prime}} \neq L^{r}$ will for sure exclude an equilibrium configuration in the shape of a pentagon, the contrary can not be stated. The cross configuration in Fig. 7 can be obtained for both the choices of $L^{r \prime}$ considered. From this point of view, excluding one of the two configurations can be seen as a method for escaping one local minimum configuration.

When considering a cross configuration as in Fig. 7, differently from the pentagon case, the potential field for 
the agent in the centre cannot be considered as for the others. Anyway it is in equilibrium whatever choice of $L^{r}$ parameter is done. This is due to the symmetry of potential acting on this agent which translates into two pairs of equal and opposite terms for the sums in (13) and (14) making both equations trivially null. For this reason the agent with $L^{r}=L^{r^{\prime}}$ will find its equilibrium position at the centre enabling the cross formation. This also justifies the consideration about the two possible arrangements for agents with the same repulsive scale distance parameter: being the central position an equilibrium one, also a group of agents with the same repulsive potential can spontaneously arrange in a cross configuration. Equilibrium for the surrounding agents according to the scheme of Fig. 7 is only determined by (13), as the $y$-component is null by symmetry. The equilibrium distance $d$ as shown in Fig. 7 is found by solving for the value $d$ that satisfies

$$
\begin{aligned}
\frac{C^{r}}{L^{r}}\left(\exp \left(\frac{-d}{L^{r}}\right)+\exp \left(\frac{-2 d}{L^{r}}\right)+\sqrt{2} \exp \left(\frac{\sqrt{2} d}{L^{r}}\right)\right)= \\
\quad \frac{C^{a}}{L^{a}}\left(\exp \left(\frac{-d}{L^{a}}\right)+\exp \left(\frac{-2 d}{L^{a}}\right)+\sqrt{2} \exp \left(\frac{-\sqrt{2} d}{L^{a}}\right)\right),
\end{aligned}
$$

which is obtained by expanding (13). As it can be seen there is no closed-form analytical solution. On the other hand, a stable equilibrium distance exists for a choice of the free parameters $C^{a}, C^{r}, L^{a}$ and $L^{r}$ satisfying the conditions stated in [19]. In particular for given $L^{r}$ and $L^{a}$, with $L^{a}>L^{r}$, a stable equilibrium can be found by tuning the parameters $C^{a}$ and $C^{r}$. This is further elaborated in Sec. III-B.

\section{B. APF Coefficient Definition}

The coefficients of the APF acting along the edges of the graph are calculated such to set the desired distance amongst the spacecraft. Just the $C^{r}$ coefficient is calculated as function of the others which are set. The change of $C^{r}$ parameter only or, more precisely, the change in the ratio $C^{r} / C^{a}$ is sufficient to modify the position of the minimum, hence the design distance, for the APF used. In particular, an interaction between two spacecraft belonging to two different $n$-agent groups is considered, with a design distance $d_{d}$; the ratio $C^{r} / C^{a}$ can hence be calculated by manipulating (19) as

$$
\frac{C^{r}}{C^{a}}=\frac{L^{r}}{L^{a}} \exp \left(d_{d} \frac{L^{a}-L^{r}}{L^{a} L^{r}}\right) .
$$

Once the coefficients are set, (22) can be reversed to calculate the equilibrium distance. When more than 2 agents are involved, an analytic expression for the equilibrium distance cannot be defined, but given a desired distance, one can always get an expression for the value of the ratio $C^{r} / C^{a}$ that produces that separation. In particular for a fully connected group of 5 agents $C^{r} / C^{a}$ ratio can be calculated equating to zero the gradient of the potential for the formation according to the scheme in Fig. 7. As the $y$-component is trivially null, $C^{r} / C^{a}$ can be calculated considering just $x$-component of the gradient in (13). This corresponds to (21), which can be manipulated algebraically to obtain

$$
\frac{C^{r}}{C^{a}}=\frac{L^{r}}{L^{a}} \frac{\exp \left(-\frac{d_{d}}{L^{a}}\right)+\exp \left(-\frac{2 d_{d}}{L^{a}}\right)+\sqrt{2} \exp \left(-\frac{\sqrt{2} d_{d}}{L^{a}}\right)}{\exp \left(-\frac{d_{d}}{L^{r}}\right)+\exp \left(-\frac{2 d_{d}}{L^{r}}\right)+\sqrt{2} \exp \left(-\frac{\sqrt{2} d_{d}}{L^{r}}\right)}
$$


This tuning method can be extended to the other links of the adjacency matrix; by defining the coefficients in this way the desired self-similar pattern is produced.

\section{Stability of Control Law}

The stability can simply be proved following a procedure similar to the one in [18]. Consider the time derivative of the energy as the sum of artificial potential and real kinetic energy,

$$
\frac{d E_{t}}{d t}=\frac{d K_{t}}{d t}+\frac{d U_{t}}{d t}
$$

where

$$
U_{t}=\frac{1}{2} \sum_{i} \sum_{j} a_{i j} U_{i j}
$$

is the total potential energy per unit mass with

$$
U_{i j}=U_{i j}^{a}+U_{i j}^{r}
$$

and

$$
K_{t}=\frac{1}{2} \sum_{i} K_{i}=\frac{1}{2} \sum_{i}\left(\mathbf{v}_{i} \cdot \mathbf{v}_{i}\right)
$$

the total kinetic energy per unit mass. Expanding (24),

$$
\frac{d E_{t}}{d t}=\sum_{i}\left(\nabla U_{t} \cdot \mathbf{v}_{i}+\frac{\partial K_{t}}{\partial \mathbf{v}_{i}} \cdot \dot{\mathbf{v}}_{i}\right)
$$

where the gradient operator $\nabla(\cdot)$ is defined in (5). Substituting (4) and (26) into (28) yields

$$
\begin{aligned}
\frac{d E_{t}}{d t} & =\sum_{i}\left[\nabla U_{t} \cdot \mathbf{v}_{i}+\frac{\partial K_{t}}{\partial \mathbf{v}_{i}} \cdot\left(-\nabla U_{i}-\sigma \mathbf{v}_{i}\right)\right] \\
\therefore \frac{d E_{t}}{d t} & =\sum_{i}\left[\left(\nabla U_{t} \cdot \mathbf{v}_{i}-\nabla U_{i} \cdot \mathbf{v}_{i}\right)-\sigma\left|\mathbf{v}_{i}\right|^{2}\right] .
\end{aligned}
$$

As the potential depends upon pairwise interactions, the derivative w.r.t. $\mathbf{x}_{i}$ is not null for both the $U_{i j}$ and $U_{j i}$ potentials that constitute the total potential $U_{t}$. If the agents interacted in a symmetric way, this would cancel out with the gradient $\nabla U_{i}$, but as the sum of the potential derivatives upon any agent includes asymmetric terms, this does not occur. Nevertheless the difference between the gradients can be always damped by the artificial viscous damping. Hence, it can be concluded that

$$
\exists \sigma>0: \sum_{i}\left[\left(\nabla U_{t} \cdot \mathbf{v}_{i}-\nabla U_{i} \cdot \mathbf{v}_{i}\right)-\sigma\left|\mathbf{v}_{i}\right|^{2}\right] \leq 0
$$

This is enabled by the fact that artificial potential and its derivative are bounded functions.

As total energy time derivative can be made a negative semi-definite function, this can be compared to a Lyapunovlike function whose derivative is always proved to be negative and zero at equilibrium, corresponding to null speed. Thus the system will leak energy and stabilise eventually into a static formation which corresponds to the minimum of total energy. 


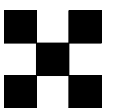

(a)

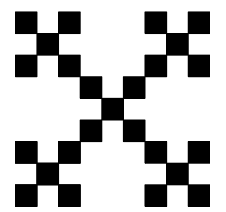

(b)

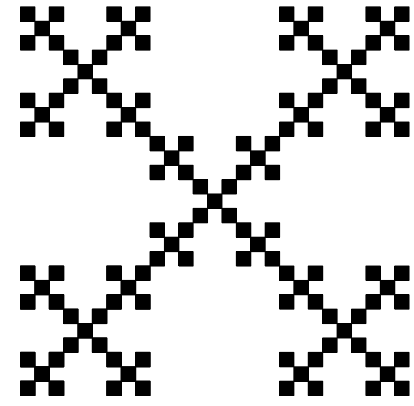

(c)

Fig. 8. First three stages of growth of the Purina fractal array for (a) $P=1$, (b) $P=2$, and (c) $P=3$.

The stability characteristic outlined above does not imply that the system will relax into the desired formation as the energy might be minimized, even just in local sense, with a configuration that is not the one the system was meant to take.

\section{Fractal Antenna Array Design and Analysis}

A distributed antenna array spread across a satellite formation offers the potential of improved directivity and gain for increasing number of elements. However, controlling a large number of satellites flying in relatively close proximity to one another does not provide a convenient solution. A more practical design would involve a formation with a reduced number of elements, that is able to achieve similar performance. Basing antenna array formations on fractal geometries provides not only the potential to reduce the number of elements but also offers the possibility to operate across a range of frequencies and the self-replicating nature of fractal patterns extends to their performance characteristics too; this means that rapid analysis of a wide range of antenna characteristics is possible.

The method described in Sec. II-C is followed here to design and analyse a planar array based on the Vicsek or Purina [15] fractal. A 3-by-3 symmetric planar array is thinned down to form the Purina fractal pattern which has the simple sub-array $\mathrm{S}_{1}$ at growth scale $P=1$,

$$
\mathrm{S}_{1}=\left[\begin{array}{lll}
1 & 0 & 1 \\
0 & 1 & 0 \\
1 & 0 & 1
\end{array}\right] .
$$

The array fractal pattern $\mathrm{S}_{P}$ at an arbitrary growth scale $P \in \mathbb{N}, P \geq 2$ is given by

$$
\mathrm{S}_{P}=\mathrm{S}_{1} \otimes \mathrm{S}_{P-1}
$$

with $\otimes$ denoting the Kronecker product, whereby a unit entry means that an element is switched on, while zero indicates that the array element is switched off, generating the entries previously discussed in (10). Fig. 8 demonstrates the first three stages of growth for the Purina fractal array. 
The array factor associated with the generating sub-array in Fig. 8(a) can be derived from (7) by setting $d_{x}=$ $d_{y}=\frac{\lambda}{2}$ with $\lambda$ the wavelength of the signal, $M=2$ and $S_{11}=1$, resulting in

$$
\Gamma_{\text {frac }}\left(u_{x}, u_{y}\right)=1+4 \cos u_{x} \cos u_{y}
$$

Substituting the array factor in (34) into (11), and with an expansion factor of $\delta=3$ relating to the size of the full square lattice array, the product form of the array factor equation at growth stage $P$,

$$
\Gamma_{P}\left(u_{x}, u_{y}\right)=\prod_{p=1}^{P}\left\{1+4 \cos \left(3^{p-1} u_{x}\right) \cos \left(3^{p-1} u_{y}\right)\right\} \quad,
$$

which is based on the simplification of (8) and (9) due to $d_{x}=d_{y}=\frac{\lambda}{2}$ to

$$
\begin{aligned}
& u_{x}=\pi\left(\sin \theta \cos \phi-\sin \theta_{0} \cos \phi_{0}\right) \\
& u_{y}=\pi\left(\sin \theta \sin \phi-\sin \theta_{0} \sin \phi_{0}\right) .
\end{aligned}
$$

With $\theta_{0}$ and $\phi_{0}$ indicating the look-direction of the beamformer, (34) can be recasted in terms of spherical coordinates $\theta$ and $\phi$. Assuming for simplicity that the look direction of the beamformer is towards broadside with $\theta_{0}=\phi_{0}=0$, the array factor

$$
\Gamma_{P}(\theta, \phi)=\prod_{p=1}^{P}\left\{1+4 \cos \left(3^{p-1} \pi \sin \theta \cos \phi\right) \cos \left(3^{p-1} \pi \sin \theta \sin \phi\right)\right\}
$$

results. Substituting (38) into (12) for isotropic sources, a reduced expression

$$
D_{P}(\theta, \phi)=\frac{\Gamma_{P}^{2}(\theta, \phi)}{\frac{1}{4 \pi} \int_{0}^{2 \pi} \int_{0}^{\pi} \Gamma_{P}^{2}(\vartheta, \varphi) \sin (\vartheta) d \vartheta d \varphi}
$$

for the directivity of the Purina array based on isotropic sources is obtained.

With the help of (39), the directivity plots for the different growth stages of the Purina fractal array in Fig. 8 can now be computed. These are shown for the first three growth stages in Fig. 9 for the case of $\phi=0^{\circ}$. In each case the directivity pattern has been normalised to its own maximum, making it possible to compare the relative performance of the various stages of growth. It can be noted that, as the number of elements increases, the gain of the main beam increases w.r.t. the sidelobe level. Also, with increasing $P$, the beamwidth decreases, i.e. the resolution of the array is enhanced. Additionally, self-similarity in the fractal array leads to self-similarity in the produced radiation pattern. Note how each stage provides an envelope for the rescaled version of the following stage.

The above steps have detailed the step-by-step procedure used in the design and analysis of a fractal array. Combining the antenna elements has the potential to alter the radiation characteristics of an ensemble of antennas and can result in a steerable and highly directive beam.

\section{Simulation Results}

The control method illustrated in this paper is used to simulate a possible operative scenario in which a spacecraft formation is used to form a distributed array in Earth orbit. A geostationary orbit - a circular Earth orbit with radius 


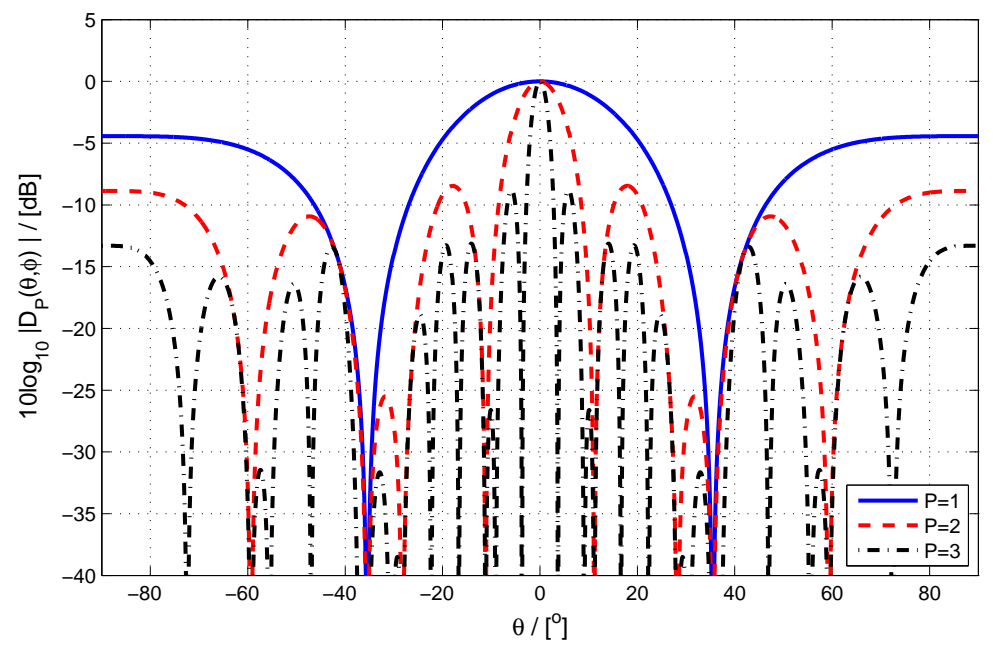

Fig. 9. Directivity plots for the first three stages of Purina fractal arrays shown in Fig. 8, with an assumed direction of the main beam towards broadside $(\theta=0)$.

$42157 \mathrm{~km}$ and 0 deg inclination - is chosen to simulate the dynamics although the application is not specifically aimed at telecommunications. Deployment of a fractal antenna array is simulated where the system is composed of 125 radiating elements.

The system requirements suggest suitable actuators and to a certain degree limit the choices regarding agent selection and separation. The method of control and the possibilities offered by reducing the size of individual radiating elements while maintaining an overall large aperture drive towards the selection of a satellite in the size range of pico- or nano-satellite suitable for a separation in the order of $1 \mathrm{~m}$. This is the separation chosen as the inter-spacecraft distance is still small enough to control motion through mutually exchanged electromagnetic forces and far apart enough to allow for relatively coarse accuracy, in particular at the release from a carrier spacecraft or launcher.

The 125 unitary mass agents reproduce the shape of a Purina fractal at a growth stage of $P=3$; they are deployed in 25 groups of 5 -agent subgroups which is the elementary unit of the formation $(N=125, n=5)$. The dynamics of the spacecraft formation is based on Clohessi-Wilthshire (CW, [21]) linearised equations in an orbiting reference frame.

The reference frame forms a Cartesian coordinate system, and is arranged such that

- the $x$-axis is tangent to the orbit and parallel to the orbital velocity vector,

- the $y$-axis is parallel to angular momentum vector, and

- the $z$-axis is orthogonal to the first two and pointing towards the Earth's centre of gravity.

The $\mathrm{CW}$ equations in this reference frame are

$$
\ddot{x}=-2 \nu \dot{z}
$$




$$
\begin{aligned}
& \ddot{y}=-\nu^{2} y \\
& \ddot{z}=-2 \nu \dot{x}-3 \nu^{2} z,
\end{aligned}
$$

where $\nu$ is the orbital frequency.

Initial conditions were set such as each spacecraft had an initial position randomly picked within a sphere centred on its final position and radius equal to 1.5 times the distance to its nearest neighbour to account for possible initial swapped positions between near agents; initial relative velocities are null. This corresponds to assuming that a carrier spacecraft or launcher releases the agents with coarse accuracy i.e. not completely random. Attitude for the single spacecraft is not considered while overall attitude control for rotation around the $x$ and $y$ axes is guaranteed by positioning control through a parabolic potential that flattens the formation onto the $x-y$ plane. Sensors are idealised, that is, the exact position of any one agent is known without delay by all the agents to which it is linked.

Although actuators are not modelled here, some characteristics relating to the possible use of electromagnetic forces are considered. In particular, actuators of the kind proposed in [22] and [23] are considered. As these actuators, particularly those based on Coulomb forces, cannot be used concurrently due to interference issues, a duty cycle is set up and the ensemble is split into a number of groups so that any two groups which are active at the same time are relatively far apart. This allows interferences to be neglected. Each group is controlled across a time period of the duty cycle. Over the whole duty cycle each group of agents is controlled for the same amount of time. As consequence, agents belonging to more than one group - e.g. linking agents between groups - are controlled for longer. The frequency of the duty cycle needs to be high enough not to allow spacecraft to drift away between control periods. This can be bounded from below by considering a linearised version of the control law and computing the frequency of the associated harmonic oscillator. Considering the APF only, the control can be linearised about the equilibrium as

$$
\begin{aligned}
m \tilde{\ddot{x}}_{i}=\sum_{j}\left\{\frac{C_{i j}^{a}}{L_{i j}^{a}} \exp \left(\frac{-d_{i j}}{L_{i j}^{a}}\right)-\frac{C_{i j}^{r}}{L_{i j}^{r}} \exp \left(\frac{-d_{i j}}{L_{i j}^{r}}\right)-\right. & {\left.\left[\frac{C_{i j}^{a}}{L_{i j}^{a}{ }^{2}} \exp \left(\frac{-d_{i j}}{L_{i j}^{a}}\right)-\frac{C_{i j}^{r}}{L_{i j}^{r}{ }^{2}} \exp \left(\frac{-d_{i j}}{L_{i j}^{r}}\right)\right]\left(x_{i}-d_{i j}\right)\right\}, }
\end{aligned}
$$

where it is assumed that the equilibrium position is at a distance $d$ from the neighbouring agents and that these agents are fixed in their positions. The sum is extended to all the neighbouring agents acting along one axis. As an example, considering the central agent of Fig. 7, this means that only 2 agents contribute to its oscillatory motion along the orthogonal axes.

Since (41) is in the form of a linearised harmonic oscillator perturbed by a constant acceleration, the frequency associated with this system is

$$
\omega_{i}=\sqrt{\sum_{j} \frac{C_{i j}^{a}}{L_{i j}^{a}{ }^{2}} \exp \left(\frac{-d_{i j}}{L_{i j}^{a}}\right)-\frac{C_{i j}^{r}}{L_{i j}^{r}} \exp \left(\frac{-d_{i j}}{L_{i j}^{r}}\right)} .
$$

Therefore, the frequency at which control is performed should not be smaller than $\sup _{i} \omega_{i}$, which is obtained by considering all the sets of values defining the control of the groups. For the case reported here, the whole duty 
TABLE I

NUMERICAL VALUES OF COEFFICIENTS USED IN NUMERICAL SIMULATIONS.

\begin{tabular}{llllll}
\hline \hline & $C^{a}$ & $C^{r}$ & $L^{a}$ & $L^{r}$ & $L^{r^{\prime}}$ \\
\hline fully connected groups (FGCs) & 4 & 3.94722 & 2 & 1 & 0.5 \\
centres of FGCs & 1 & 0.99596 & 4.5 & 4 & 2 \\
periph. agents betw. adjacent FGCs & 0.8925 & 1 & 2 & 0.5 & \\
centres of 25-agent groups & 2500 & 2505.3 & 10 & 9.9 & 4.5 \\
peripheral of 25-agent groups & 69.96 & 70 & 3 & 2.9 & \\
\hline$\sigma=0.1$ for all the agents & & & & & \\
\hline \hline
\end{tabular}

cycle lasts 2 seconds and the 125 spacecraft are considered as belonging to 9 groups, which are

- the 5 -agent groups at the centre of the 25 -agent groups,

- the 5 -agent groups at the top of the 25-agent groups,

- the 5 -agent groups at the bottom of the 25 -agent groups,

- the 5 -agent groups at the left of the 25-agent groups,

- the 5 -agent groups at the right of the 25 -agent groups,

- the agents linking the centres of the 5-agent groups in the 25-agent groups,

- the agents bonding the 5 -agent side by side in the 25 -agent groups,

- the agents bonding the centres of the 25 -agent groups, and

- the agents bonding the sides of the 25 -agent groups.

The connections between each group (consisting of 25 agents) are ensured by pairs of agents instead of groups of agents. This allows a reduction of the computational efforts for each agent and a reduction of the computational resources needed for the simulation. On the other hand this reduces the control power and slows down the deployment of the formation. Tab. I shows the values of the coefficients used.

The agent at the centre of the formation (say agent 1) is the only one linked to the centre of the reference frame by a quadratic potential in the form $U_{c}=\zeta\left|\mathbf{x}_{1}\right|^{2}$, with $\zeta=0.1$ as a weighting parameter. This is to provide a kind of orbit tracking capability or, in practical terms, the possibility to stay anchored to the centre of the reference frame. This also suggests the task of tracking the orbit can potentially be carried out by a single agent only, while the others just track their relative position with respect to the central agent. Without loss of generality, for simplicity here the central agent is assumed to track the orbit. The control law is applied for just $x$ and $y$ axes of the orbital reference frame with control on $z$-axis performed through a simple parabolic potential $U_{z i}=\zeta\left|\mathbf{z}_{i}\right|^{2}$, for $i=1 \ldots N$, that flattens the formation on the plane $z=0$, where again $\zeta=0.1$ is a weighting parameter. The actions of both the quadratic potentials are damped by virtual dissipative terms $\sigma \dot{\mathbf{x}}_{i}$.

Snapshots from the deployment are shown in Fig. 10. It can be noted that after one day the deployment exhibits slight distortions in particular within peripheral groups. 
Finally in Fig. 11 errors on the designed relative position after one day are plotted. The error measure is the difference between the actual distance of each spacecraft from the centre of the formation and the ideal design distance; this is then plotted as a percentage of the desired spacing. It can be seen that the maximum error is lower than 5\%. The evaluations of both the snapshots in Fig. 10 and the error in Fig. 11 are considered after a maximum of 24 hours; this is sufficient to prove the self arranging capabilities of the control technique. After a further 24 hours the magnitude of the maximum error is halved as compared to the $24 \mathrm{~h}$ values in Fig. 11. Theoretically a complete relaxation with no positioning errors is possible but only after an infinite period of time due to the viscous-like damping.

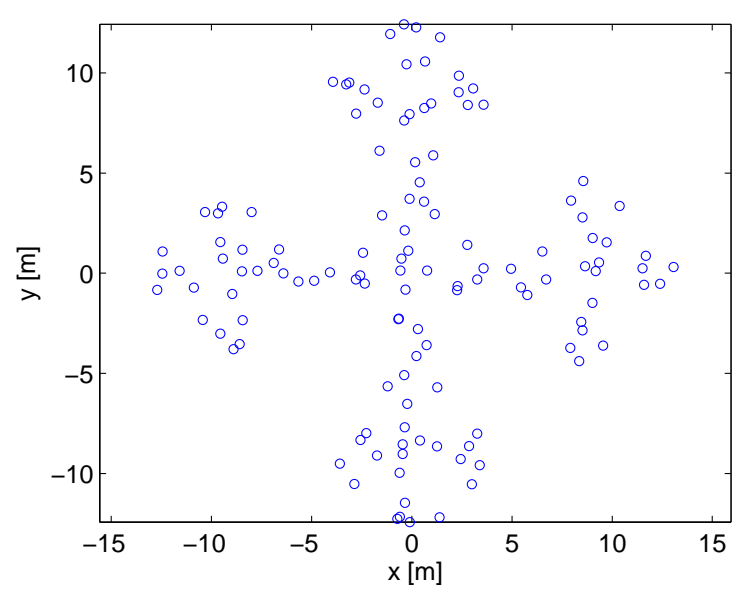

(a)

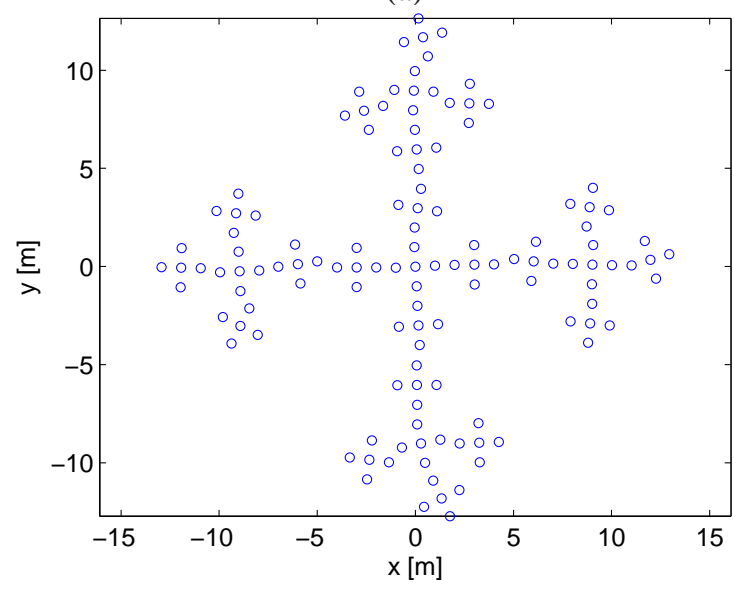

(c)

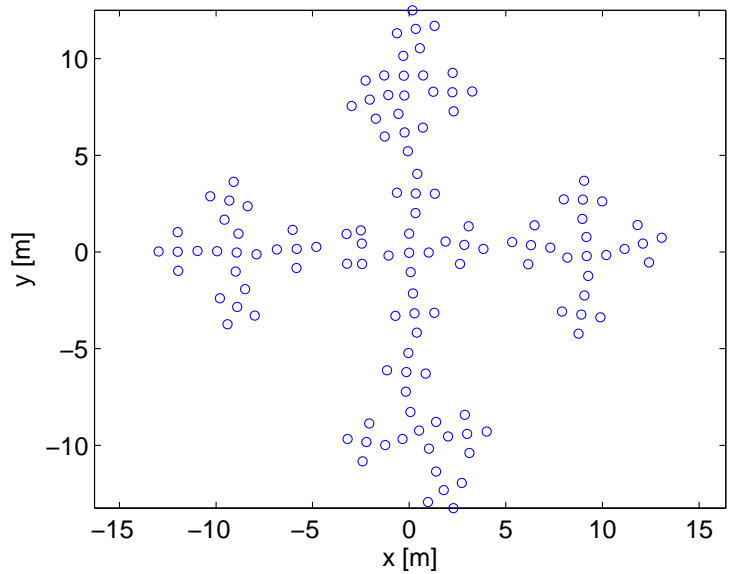

(b)

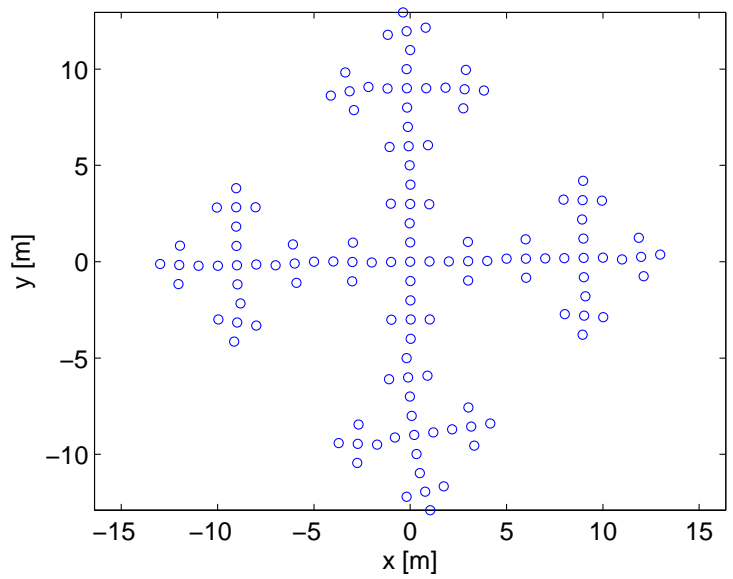

(d)

Fig. 10. Formation deployment in GEO, with snapshots taken at (a) $t=0 \mathrm{~s}$, (b) $t=60 \mathrm{~s}$, (c) $t=3600 \mathrm{~s}=1 \mathrm{~h}$, and (d) $t=86400 \mathrm{~s}=24 \mathrm{~h}$.

\section{Discussion}

The idea of meeting needs for highly directional antenna arrays through a space based fractionated architecture is constructed around the possibility of locating a number of spacecraft, each carrying an antenna element, according 


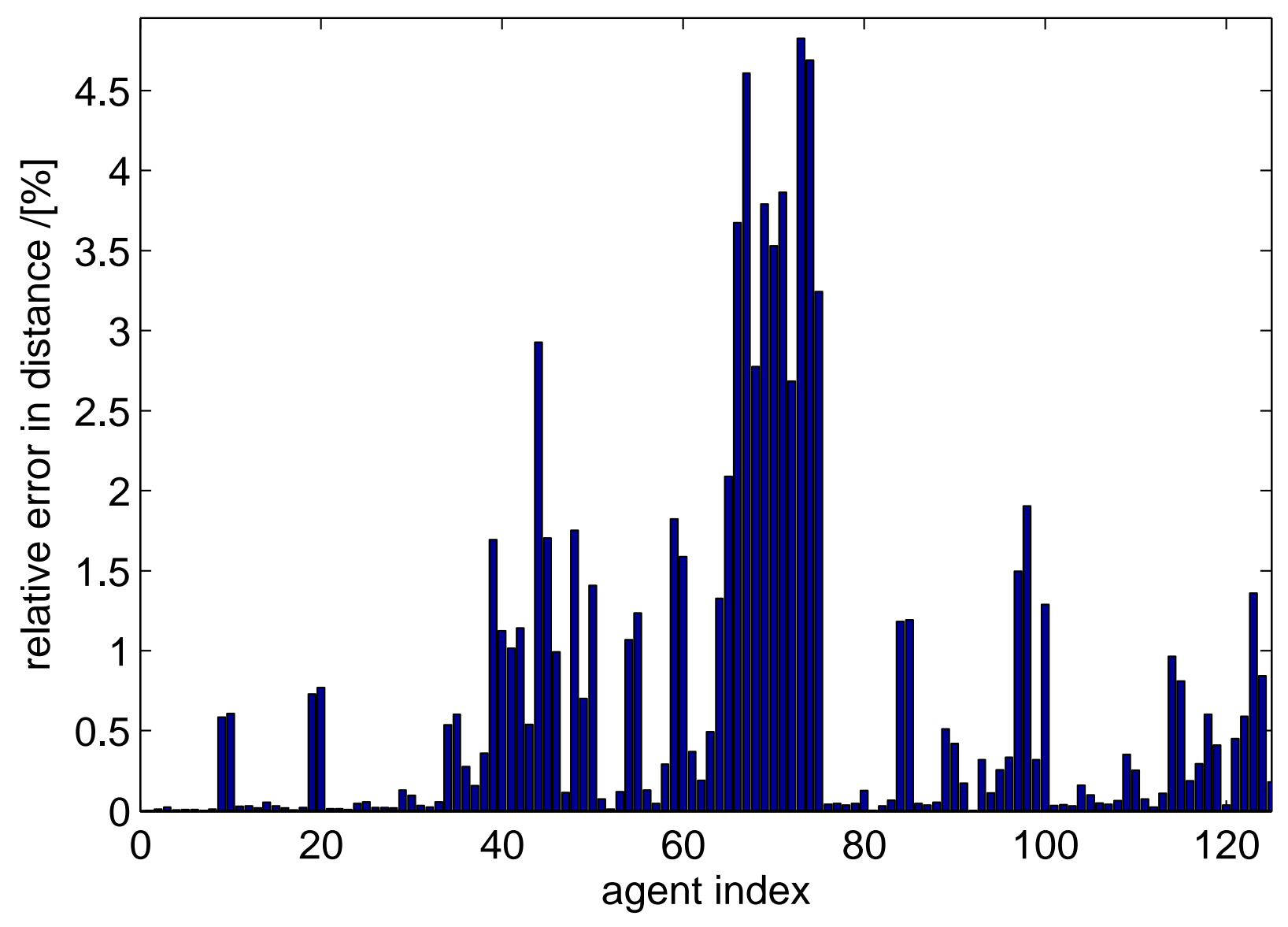

Fig. 11. Errors in relative design positioning after 1 day from release of the formation. Distances are computed with respect to the agent at the centre of the formation, and the distance error expressed as a percentage of the ideal design distance.

to a precise fractal scheme. This improves overall antenna performance and capabilities while using a contained number of elements. In turn the possibility of using small spacecraft enables the formation of a fractionated antenna, but requires accurate spacing between the elements. Orientation is not considered here for single agents as they are assumed to be isotropic sources. Thus, in the case of an antenna array as described above, the relative agent positions within the whole array is the key requirement as this influences the performance of the array. Hence considering just coarse attitude control for single agents, a description of the system characteristics in a global sense is possible as long as relative positions are precisely known. Utilising this knowledge, directivity through array phasing is achievable at group level for compensation of global attitude errors and at agent level to accommodate misalignment of the single elements.

From a control point of view the need for precise close formation flying can be tackled through using reliable techniques and implementing these on relatively small agents. In this respect, artificial potential functions are particularly suited for the task as their stability characteristics are analytically provable, hence they do not need extensive Montecarlo test campaigns to validate their behaviour. Moreover, APFs allow for highly non-linear control 
through quite straightforward computation due to their smoothness. As the amount of information needed is just the relative position of a number of neighbours, the connection network presented here has the double advantage of shaping the formation on one side and reducing the number of connections on the other. These combined characteristics make small spacecraft, even with reduced computation capabilities, able to carry out the task of arranging into a formation through exclusively inter agent interaction in a decentralised way.

The artificial potential functions account for collision avoidance of the spacecraft as long as they are connected in the network, which holds for any two spacecraft whose nominal positions are in close proximity. Two agents may then collide if they are in close proximity while they are not meant to be, hence there is not a connection between them. This is anyway avoided by choosing the initial conditions adequately, that is collocating each spacecraft within its basin of attraction with an initial velocity within the control capability of the actuators. This also accounts for the problem of local minima which are typical of APF control methods. It would be possible to account for collision of non-communicating spacecraft by triggering avoidance manoeuvres in case of closeness revealed by any sensor scanning of the local neighbours. These kinds of avoidance manoeuvres are to be designed not to introduce persistent instability in the control of the agents already linked through the network and their analysis is beyond the scope of this work.

Although the paper is not focussed on the dynamics of the formation in the orbit environment, the definition of the simulation scenario imposes to consider specific orbit parameters and suitable actuators. Here, a geostationary orbit was considered although agents are not specifically targeted at telecommunication purposes. When dealing with actuator modelling, it was decided to keep the topic as close as possible to one of control, that is, actuator characteristics were considered only in part. Although the response of the actuators was not included, their choice took into account the close proximity scenario and the use of inter-agent electromagnetic forces was proposed rather than thrusters, which may imply plume impingement problems. Moreover the APF methods drive the system through an oscillatory stage before the achievement of the equilibrium configuration during which residual energy (both virtual potential and real kinetic) is dissipated. This translates into fuel wasting when considering the use of thrusters. The introduction of a duty cycle in the control operation is a consequence of the choice of actuators. Another advantage of having actuators that mimic the virtual inter-agent action of the artificial potential makes the analysis applicable to a wider selection of possible actuators. The duty cycle just applies to inter agent actions for which Coulomb forces can be considered. Indeed in [24] and [22] it was shown how a closely spaced formation can be maintained in GEO orbit using this type of actuation. For what concerns the $z$-axis, the use of Lorentz forces as in [23] might be considered, although their effectiveness is to be investigated further in relation to the magnetic environment.

The communication network was intended in the first place for control purposes only, but the need for task assignment in the fractionated architecture as well as array phasing can be carried out through the same architecture. In particular, the system inherits a structured hierarchical network, where the ranking of the agents depends on the number of links they are connected to. This does not imply that the resulting architecture is centralised, but allows the task assignment to be carried out on the basis of the hierarchy of the agents. For instance the guidance for the 
whole formation can be carried out by a number of spacecraft which communicate in an all-to-all scheme in order to share the computational efforts (e.g. the centres of the 25-agent groups), and then passed to another module able to compare this to the navigation to eventually generate a control input for the whole formation. This is different from the guidance, navigation and control functions that each spacecraft carries out: while each spacecraft should find its position in a distributed architecture, the whole system follows a guidance law that enables the mission task achievement. It is worthwhile stressing how the position of each agent is not pre-determined in a strict sense. The links of each agent are pre-assigned, but this does not prevent agents, or groups of agents belonging to the same level to swap their positions.

A final consideration about the planarity of the formation can be done. The main claim of this paper, for what concerns the control part, is to propose a control architecture that exploits emergent behaviour shaped by the connection network. It was considered that a $2 \mathrm{D}$ application is sufficient to prove the main feature of the technique. Nevertheless the same considerations about the emergence of a central symmetry and the building up of several hierarchical levels in a self-similar fashion can be applied to 3D formations as well as an initiator composed of a different number of agents.

\section{CONCLUSIONS}

In this paper the deployment of a self-similar formation of autonomous agents aimed at producing a fractal geometry array was for the first time investigated in the context of a space-based distributed antenna array. Artificial potential functions and self-similar adjacency matrices were used to obtain self-similar patterns in a formation of mobile agents, while electrodynamic analysis was used to assess the performance and potential benefits that arise from the fractal patterns. The formation deployment was simulated in geostationary Earth orbit, and demonstrated the feasibility of the concept.

The exploitation of emergent self-similar, or fractal, patterns in space-based antenna arrays is encouraged by the reduced sensitivity of the performances of the array to element failure, and by the possibility to account for positioning errors through actively controlling the phasing of the array elements. Moreover the fractal geometry of the array allows for performances in terms of directivity that are comparable, or even improved, to that of a classical square lattice scheme which makes use of a higher number of elements.

The APF method enables the use of analytic tools to draw the characteristics of the control law in terms of the stability and achievement of final desired configuration. The self-similar connection scheme used accounts for multiple redundancy towards dispersion, that is any link between two agents can be lost without catastrophic consequences for the whole formation. The system is cooled-down using artificial damping which, in terms of control, represents an improvable means as the dissipation of artificial potential energy may translate into real fuel waste for the actual agents. The aim of avoiding undesirable effects due to the choice of thrusters as actuators drove towards considering electromagnetic inter-agent forces to control the formation for simulation purposes in GEO environment.

Finally, the use of multiple independent elements to form the array allows for relaxation of attitude control 
requirements for the single agents, shifting from an attitude problem to one of relative agent/group positioning that defines the attitude for the whole formation.

\section{REFERENCES}

[1] J. Leitner, "Formation flying: The future of remote sensing from space", NASA, Goddard Space Flight Center, MD, United States, Tech. Rep., 2004.

[2] E.A. Barnes and B.E. Bishop, "Utilizing navigational capability to control cooperating robotic swarms in reconnaissance- based operations", Proceedings of the 40th Southeastern Symposium on System Theory, pp. 338-342, March 2008.

[3] S. Schwartz, "Cross-scale - multi-scale coupling in space plasmas", ESA, Tech. Rep., December 2009.

[4] M. Bester, M. Lewis, B. Roberts, J. McDonald, D. Pease, J. Thorsness, S. Frey, D. Cosgrove, and D. Rummel, "Themis operations”, Space Science Reviews, vol. 141, no. 1-4, pp. 91-115, December 2008.

[5] P.R. Lawson, O.P. Lay, S.R. Martin, C.A. Beichman, K.J. Johnston, W.C. Danchi, R.O. Gappinger, S.L. Hunyadi, A. Ksendzov, B. Mennesson, R.D. Peters, D.P. Scharf, E. Serabyn, and S. C. Unwin, "Terrestrial planet finder interferometer 2006-2007 progress and plans - art. no. 669308", Techniques and Instumentation for Detection of Exoplanets III, vol. 6693, pp. 69308-69308, September 2007.

[6] K. Danzmann, "Lisa - an esa cornerstone mission for the detection and observation of gravitational waves", Advances in Space Research, vol. 32, no. 7, pp. 1233 - 1242, 2003.

[7] F. Sjoberg, A. Karlsson, and B. Jakobsson, "Proba-3: A formation flying demonstration mission on the verge to be realised", Proceedings of the 3rd International Symposium on Formation Flying, Missions and Technologies, ESA-ESTEC, (Special Publication) ESA SP, no. 654 SP, April 2008.

[8] G. Blackwood, O. Lay, A. Deininger, W. Ahmed, R. Duren, C. Noecker, and B. Barden, "The starlight mission: A formation-flying stellar interferometer", Proceedings of SPIE - The International Society for Optical Engineering, vol. 4852, no. 2, pp. 463 - 480, February 2002.

[9] C.R. McInnes, "Velocity field path-planning for single and multiple unmanned aerial vehicles", Aeronautical Journal, vol. 107, no. 1073, pp. 419-426, July 2003.

[10] C. Balanis, Antenna theory: analysis and design. Wiley, New York, 1997.

[11] D.H. Johnson, Array signal processing:concepts and techniques. Prentice Hall, Englewood Cliffs, NJ, 1993.

[12] M.J. Bentum, C.J.M. Verhoeven, and A.J. Boonstra, "OLFAR - orbiting low frequency antennas for radio astronomy", in Proceedings of the ProRISC 2009, Annual Workshop on Circuits, Systems and Signal Processing, Veldhoven, pp. 1-6, November 2009.

[13] M. Ayre, I. Izzo, and L. Pettazzi, "Self assembly in space using behaviour based intelligent components", in Proceedings of the Towards Autonomous Robotic Systems: Incorporating the Autumn Biro-Net Symposium, Imperial College, London, United Kingdom, September 2005.

[14] D. Izzo and L. Pettazzi, "Autonomous and distributed motion planning for satellite swarm”, Journal of Guidance, Control, and Dynamics, vol. 30, no. 2, pp. 449-459, March/April 2007.

[15] D.H. Werner and R. Mittra, "The theory and design of fractal antenna arrays", in Frontiers in Electromagnetics, 1st ed. Wiley-IEEE Press, Chap. 3, pp. 94-203, 2000.

[16] C. Puente-Baliarda and R. Pous, "Fractal design of multiband and low side-lobe arrays", Antennas and Propagation, IEEE Transactions on, vol. 44, no. 5, pp. 730-739, May 1996.

[17] D.J. Bennet and C.R. McInnes, "Verifiable control of a swarm of unmanned aerial vehicles", Proceedings of the Institution of Mechanical Engineers, Part G: Journal of Aerospace Engineering, vol. 223, no. 7, pp. 939-953, November 2009.

[18] C.R. McInnes, "Vortex formation in swarms of interacting particles", Physical Review E (Statistical, Nonlinear, and Soft Matter Physics), vol. 75, no. 3, March 2007.

[19] M.R. D’Orsogna, Y.L. Chuang, A.L. Bertozzi, and L.S. Chayes, "Self-propelled particles with soft-core interactions: Patterns, stability, and collapse", Physical Review Letters, vol. 96, no. 10, pp. 1-4, March 2006.

[20] A. Maffett, "Array factors with nonuniform spacing parameter", IRE Transactions on Antennas and Propagation, vol. 10, no. 2, pp. 131 -136, March 1962

[21] H. Schaub and J. Junkins, Analytical mechanics of space systems. Reston, Va.: American Institute of Aeronautics and Astronautics, 2003. 
[22] L. King, G. Parker, S. Deshmukh, and J. Chong, "Study of interspacecraft coulomb forces and implications for formation flying", Journal of Propulsion and Power, vol. 19, no. 3, pp. 497 - 505, May/June 2003.

[23] M.A. Peck, B. Streetman, C.M. Saaj, and V. Lappas, "Spacececraft formation flying using lorentz forces", Journal of the British Interplanetary Society, vol. 60, pp. 263-267, 2007.

[24] J. Berryman and H. Schaub, "Static equilibrium configurations in geo coulomb spacecraft formations", in Advances in the Astronautical Sciences/ American Institute of Aeronautics and Astronautics, Copper Mountain, CO, United states, pp. 51-68, January 2005.

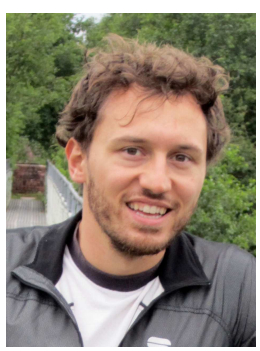

Giuliano Punzo is a Research Assistant at the department of Mechanical and Aerospace ESngineering of the University of Strathclyde. He got his BEng and MEng at Napoli University - Federico II. He worked one year with Thales Alenia Space in Turin developing technologies for space exploration and joined the Advanced Space company laboratory as $\mathrm{PhD}$ student in 2009. His research interests include distributed control and the dynamics of swarm systems.

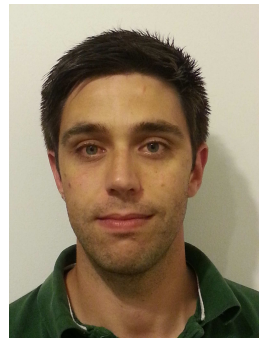

Philippos Karagiannakis received a MEng degree (with distinction) in Electronic and Electrical Engineering, from The University of Strathclyde, in 2009. Currently, he is working towards the Ph.D. degree under the supervision of Dr. Stephan Weiss at The University of Strahclyde,the focus of his research is communications for nano-satellite networks. His research interests include adaptive signal processing, large scale sparse array and distributed processing techniques.

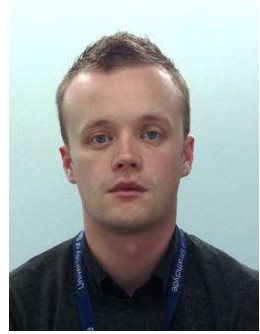

Derek J. Bennet is a research associate in the Advanced Space Concepts Laboratory within the Department of Mechanical and Aerospace Engineering at the University of Strathclyde. He has previously worked as a visiting research fellow in Japan at Tokyo Institute of Technology (2005) and Nihon University (2009) and is a chartered engineer (CEng MIMechE). 


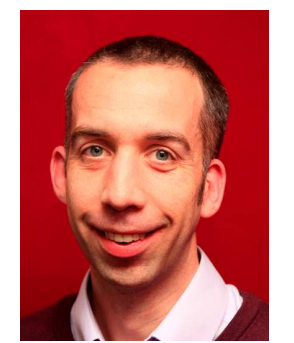

Malcolm Macdonald obtained a B.Eng in aeronautical engineering from the University of Glasgow in 2000. He then studied for his $\mathrm{PhD}$ at the University of Glasgow from 2000 - 2002, prior to gaining a research assistant post until December 2004. From 2005 - 2008 he worked at SciSys UK Ltd. on a range of European Space Agency missions throughout the project life cycle, progressing to become a senior member of technical staff leading a team working across non-linear dynamic systems and control, modelling and simulation and advanced mission and concept studies. Since 2008, he has been an Associate Director of the Advanced Space Concepts Laboratory at the University of Strathclyde, where his research interests cover celestial mechanics, swarming systems, mission analysis and design, spacecraft systems, including solar sailing and nano-spacecraft, and unmanned autonomous systems.

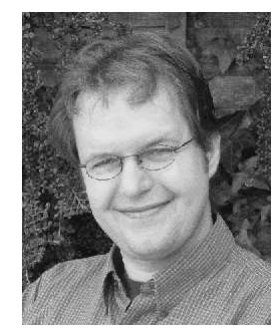

Stephan Weiss received a Dipl.-Ing. degree from the University of Erlangen-Nürnberg, Erlangen, Germany, in 1995, and a Ph.D. degree from the University of Strathclyde, Glasgow, Scotland, in 1998, both in electronic and electrical engineering. He is currently a Reader in the Department of EEE at Strathclyde. From 1999 until 2006, he held lecturer and senior lecturer positions within the School of Electronics and Computer Science at the University of Southampton. Prior to this, he was a Visiting Lecturer at the University of Strathclyde in 1998/99 and a Visiting Scholar at the University of Southern California in 1996/97. His research interests lie in adaptive, multirate, and array signal processing with applications in communications, audio, and biomedical signal processing, where he has published more than 200 technical papers. For his work in biomedical signal processing, he was a co-recipient of the 2001 research award of the German society on hearing aids. In 2011 he was a co-recipient of the VTC-Spring best paper award in the MIMO systems track. Dr Weiss is a member of EURASIP and a senior member of the IEEE. 